temporánea, Madrid, Cincel, 1990. [Introducción a conceptos y autores.]

ORnz-Oses, A.: Metafisica del sentido, Bilbao, Universidad de Deusto, 1989. CCalificada por E. Trías -El País (10-II-1991) - como una warqueología del sentido». Metafísica simbolica poseranosiana.]

Ross, W. (ed): Nuestro imaginario cultural, Barcelona, Anthropos, 1992. (Eplogo sobre la hermeneutica imaginal.)

ScHokel, Luis Alonso: Hemenéutica de la palabra, Madrid, Cristiandad, 1987. [Tratado de hemenéutica bíbica (católica).]
Sobre hemenéutica. Monográfico de Estudios filosoficos, 95 (1985).

VATnMo, G. (ed.): En tomo a la posmodemidad, Barcelona, Anthropos, 1990. [Autores varios sobre la poshermencutica.]

Veruat, A. (ed.): El retomo de Hemes, Barcelona, Anthropos, 1989. [Simposio sobre Initocritica y herneneutica simbolica.]

VV.AA.: Poetica del imaginorio. Simposio semiotico-hemenétutico-mitocrtico, Universidad de Sevilla, Facultad CC. Información, 1990.

\title{
La identidad en conflicto
}

\author{
CARLOS PEREDA \\ UNAM, México
}

Algunas preguntas de interés en relación con la identidad de los seres humanos se formulan interrogando "cquièn soy yo?", "¿quién eres tú?". ¿Qué decir de estas preguntas, además de que son prestigiosas?

En primer lugar, se trata de preguntas referenciales cuyas respuestas poseen la forma general "yo soy un $x$, wtú eres un $x \%$. Responder es satisfacer la variable introduciendo diversos conceptos biológicos, sociales, psicológicos. Por ejemplo, algunas respuestas son: yo soy un hombre de tez blanca, un esposo, un padre, un profesor de filosofía de la UNAM, un ciudadano de clase media, un habitante de la ciudad de México, un melancólico, un aprensivo... Las listas de referencias, tanto las que corresponden al yo como al tú, son ilimitadas y pueden incluir una gran variedad de las clasificaciones biologicas, sociales y psicológicas que pone a disposición una tradición. Sin embargo, esas respuestas pocas veces se dan como predicaciones aisladas a la manera de "yo soy un hombre de tez blanca", "un esposon, "um padre*... Cuando se nos pregunta o preguntamos quién soy yo, quién eres tú, la respuesta tiende a procurar que las diversas predicaciones se articulen en narrativas. Ni yo ni tú somos acumulaciones desordenadas de predicaciones biológicas, sociales, psicológicas. Cada uno de nosotros es una narrativa singularísima que va totalizando de cierta manera las varias atribuciones. Yo soy..., tú eres... una existencia, una historia concreta. $A$ esas narrativas particulares, a esas historias específicas que yo soy y que tú eres las llamo uidentidad material" de los seres humanos. A las narativas que conforman esta identidad se las puede distinguír, a su vez, en dos grandes grupos: aquellas con predicaciones que atañen a los seres humanos en tanto individuos - Pedro vivió en Tampi$c 0$, Pedro es un padre...- o identidad individual; y aquellas otras con predicaciones que lo ubican en tanto miembro de un grupo significativo - los negros, las mujeres, los homosexuales, los latinoamericanos... - o identidad grupal: la identidad grupal se articula a partir de experiencias con ciertos prototipos socia- 
les, sus expectativas, sus sanciones, sus narrativas. Por supuesto, la distinción entre identidad individual y grupal es relativa: depende de lo que, de caso en caso, se considere como "grupo significativo". Pero regresemos a nuestras preguntas.

En segundo lugar, no hay que olvidar que preguntas como "¿quién soy yo? ${ }_{1}$ "¿quién eres tú?», además de referenciales, son críticas. Sean las historias que fueren ser yo o tú, esas historias que yo soy o que tú eres tendrán la capacidad de autorreferirse críticamente. La pregunta "¿quién soy yo?" contiene de manera implícita la pregunta «quién soy yo realmente?", y lo mismo con la pregunta "¿qquién eres tú?". Cuidado, en relación con ese "realmente", atiéndase al hecho de que puedo renunciar a cada wna de las predicaciones que conforman esa historia que es mi identidad material, sin por eso tener que renunciar a mi identidad. Asî, obsérvese que puedo imaginar que ha cambiado $\mathrm{mi}$ tez blanca, que me he divorciado, que he perdido a mis hijos, que me han echado de la UNAM, que me he empobrecido, que ya vivo en otra ciudad, que he perdido mi melancolia y mis aprensiones... sin pensar que he dejado de ser quien soy. Pero, entonces ¿quiénes son ese yo y ese tú que pueden, en principio, al parecer, renunciar a todo lo que son sin dejar de ser quienes son? La vieja respuesta es, claro: yo y tú somos, antes que nada, personas. Me pueden quitar, puedo perder o renunciar a cualquier fragmento de esa historia que soy, mientras conserve mi humanidad, no dejaré de ser quien soy. Diré que esta respuesta -que invita a bastantes preguntas, por cierto- introduce la "identidad formal" de un ser humano. "Identidad formal» es otra manera de hablar prácticamente de los seres humanos, esto es, de hablar de ellos en tanto personas. Así, a partir de su identidad formal, un individuo de la especie humana, además de pertenecer a la clase natural e histórica "ser humano", pertenece a la clase normativa "persona"."

Pero ¿qué se propone cuando se distingue entre un sentido material y otro formal de identidad? No se comienza a elaborar una lógica o una metafísica de la identidad, sino algo así como una teoria de la próctica de la identidad no tradicional: antropologías, morales y hasta políticas de la identidad en sociedades no tradicionales. En efecto, ambos sentidos son conceptos clasificativos, pero, ante todo, instrumentos de identificación, identificadores de mí mismo, identificadores de los otros.

Muchas son las perplejidades, los conflictos y los problemas en tomo a estos sentidos, en general, en relación con los identificadores personales. En lo que sigue me detendré -aunque no lo suficientemente- sólo en dos dificultades: primero, cómo se relacionan entre sí los sentidos de identidad material, sobre todo grupal, y de identidad formal y, segundo, cuáles son algunos de los pros y los contras que conllevan ambos sentidos.

Exploremos tres posibles relaciones entre los sentidos de identidad material y formal con respecto a los seres humanos: compatibilidad, rivalidad simple o escisión y rivalidad compleja o desgarramiento.

Por lo pronto, sospecho que con frecuencia, por decirlo así, en los casos interesantes, no hay mera compatibilidad entre los sentidos de identidad material y formal, sobre todo entre los sentidos de identidad material grupal e identidad formal. Más todavía, solemos juzgar em-

* Teoricamente, la expresión sidentidad formal conforma otra manera de referirse al kyo trascendental*. 
pleando al uno en contra del otro. Recuérdense protestas del tipo: "soy una secretaria, pero también soy una persona, por eso tráteme como tal». En expresiones como ésta se busca que la identidad formal prime sobre la identidad material.

Un ingeniero o un arquitecto podrán actuar muy eficazmente al construir un campo de concentración, sus identidades materiales en tanto arquitectos o ingenieros podrán, así, estar en paz, pero no su identidad formal como personas. En general, es posible frente a cualquier profesión juzgarla de acuerdo a una doble identidad y este juicio es radicalmente diferente, pues no es lo mismo acusar a alguien de inepto que acusarlo de inhumano; se puede ser lo uno sin ser lo otro y viceversa. Una situación clásica que articula a esta identidad en conflicto la encontramos en la escena 1 del Acto II de ese himno a la Ilustración que es La flauta mágica. Uno de los personajes indica: "... Piensa: él es un principes. A lo cual, otro personaje le replica: "Mas todavia! El es un hombre".

A partir de estos ejemplos parece sugerirse que en cualquier conflicto entre la identidad material y la identidad formal de un ser humano hay que preferir su identidad formal. Si este fuera el caso, nos encontrariamos ante una rivalidad simple: ante un concepto escindido. De esta manera, el concepto de identidad estaria, de hecho, muchas veces escindido entre un sentido material y otro formal, aunque siempre tendríamos más argumentos a favor del sentido formal. Esto es, cualquiera sea el debate en que la identidad entrara en conflicto entre sus sentidos material y formal, sabriamos a priori que habrá siempre más argumentos para defender su sentido formal.

Casi diría, lamentablemente, este no es el caso; sin duda, ha habido, hay y probablemente seguirá habiendo muchas discusiones de interés en las que, por lo menos, tienen un peso similar los argumentos para defender tanto un sentido de identidad como el otro, en particular en lo que se refiere a la identidad grupal y a la identidad formal. Por eso, el concepto de identidad no está meramente escindido, sino desgarrado, genuinamente desgarrado e incluso desgarrado entre dos tradiciones. ¿Cómo es esto?

Hablaré de una "tradición de lo sublimen que defiende al concepto de una identidad formal como prioritario y hasta exclusivo. "Identidad formal», esto es, identidad en tanto persona, y "tradición de lo sublime" son expresiones que entre los modernos aluden al optimismo liberal y, desde el punto de vista teórico, casi inevitablemente a un nombre propio: «Kant». A la pregunta "¿quién soy yo?", Kant responde: en el sentido más relevante, yo y tú somos cualquiera. Esto es, moral o políticamente, cada ser humano cuenta y posee el mismo valor que todos los otros seres humanos sin importar para nada su «identidad material\%. Recuérdese la primera fómula del imperativo categórico: "actúa de tal manera que puedas querer que la máxima de tu acción se convierta en ley universal». Que es otra forma de decir: actúa de tal manera que cualquiera podría querer actuar de la misma manera.

Recuérdese también la segunda fórmula del imperativo: sactúa de tal manera que uses la humanidad tanto en tu persona como en la persona de los demás siempre como fin y nunca meramente como medio". Esta "humanidad", que poseen en común tanto mi persona como las demás personas y que nunca hay que usar meramente como medio, es nuestra «identidad formals. Precisamente, es esa identidad formal el soporte de todos los deberes y de todos los derechos, tanto morales como políti$\cos$, y la medida para evaluar si éstos se cumplen o no. Lo demás, las vicisitudes de nuestras historias individuales o sociales, el laberinto de las «identidades 
materiales", no es más que la situación accidental en la que nos encontramos para llegar a realizar la «identidad formal", que es universal, y que es lo que cuenta. No sorprenderá entonces que, desde esta perspectiva, los problemas de la práctica de la identidad no tradicional de la doble identificación —cómo me identifico, cómo identifico a los otros- casi no existan, o por lo menos sean muy fáciles de resolver. Las respuestas a las preguntas "¿quién soy yo?» y "¿quién eres tú?» son simples: tú y yo somos personas. La trabajosa individuación en sociedades y biografias es un hecho, pero un hecho moral y políticamente secundario. Lo primero, lo que importa: común a todas las personas es la misma identidad formal.

No pocos, sin embargo, han protestado: estas respuestas simples de la tradición de lo sublime son, en realidad, simplificaciones indebidas, simplezas engañosas y hasta sintomáticamente engañosas: muestran que se ha sucumbido en verrtigos simplificadores. Así, en contra de esta tradición y de su propuesta, una identidad formal en tanto identificador universal de los seres humanos, hay que evocar varias críticas desde posiciones contextualistas que defienden ciertas identidades grupales, y que es posible ubicar como conformando una «tradición de la bajeza». Pertenecen a ella una serie de desconfianzas y sospechas sobre la abstracción que lleva consigo la propuesta de cualquier identificador universal. Considenemos los siguientes dos ataques.

Primer ataque: este identificador sólo conforma una méscara en tanto ayuda a tapar miserias bien precisas, "bajezas" que es necesario atender, so pena de hacernos cómplices de universalidades injustas, a la vez infatuadas y llenas de peligro.

Segundo ataque: las ventajas de una identificación tan simple son ilusorias. Identificarse como persona e identificar a los otros meramente como personas es acaso la forma más pobre, más vacía, de identificarse, de identificar.

Durante el siglo XIX y en gran parte del $\mathrm{xx}$, estos dos ataques —el identificador universal es encubridor, el identificador universal es vacío- tuvieron representantes importantes en los teóricos del movimiento obnero. Se aduce: insistir en que los obreros y los patronos son personas es distraer la atención acerca de lo que más importa con respecto a ellos, sus diferencias. Son estas diferencias lo que hay que pensar, y desde estas diferencias; de lo contrario, nos perdemos en fantasías que, mediante abstracciones, falsean por completo las realidades a que, en vano, se pretende aludir. Además, identificar e identificarse en tanto persona no permite crear esos vínculos de reconocimiento tan necesarios para la accion que constituyen la "solidaridad". Porque mientras hay algo así como una «solidaridad obrera, hablar de una «solidaridad humanas no es más que vaga retórica, propicia a sentimentalismo y declaraciones oficiales, pero poco efectiva.

Argumentos con algún grado de similitud se han dado en relación con la gente de color, con las mujeres, con los homosexuales, con las diversas etnias y hasta con áreas culturales enteras como América Latina. Así, se puede hablar de la identidad - grupal, por supuesto- de los negros, de las mujeres, de los homosexuales, de las diversas etnias y hasta de América Latina, o de las varias identidades nacionales que la componen; por ejemplo, hay un feminismo radical, a veces llamado efeminismo de la diferencian, que insiste en que el feminismo es algo más que una lucha para conquistar los derechos de las mujeres: es una "visión del mundon basada en la experiencia de vivir bajo condiciones distintivas, "visión" que procura recoger todo lo aprendido en la cultura femenina tradicional y en sus valores, como un medio de contrarrestar la opresiva cultura mas- 
culina. También encontramos afirmaciones como éstas en discusiones sobre los negros, sobre los homosexuales y sobre América Latina, fragmento de geografía, de historia y de culturas a las que incluso se les atribuye un ethos particular, hasta un "destino", En cualquier caso, indagar las identidades materiales lleva a menudo a concluir que la verdadera preocupación no atañe a la identidad, sino a las diferencias, el plural de diferencias que me constituyen y constituyen a los otros, a los diversos grupos sociales y sus respectivas pertenencias, reconocimientos y solidaridades.

¿Quê decir de estos ataques contextualistas al identificador formal?

2

Para comparar algunos pros y algunos contras de usar los sentidos material y formal de identidad hay que distinguir, por lo menos, entre los usos explicativos y normativos de estos sentidos.

Los sentidos materiales pueden tener usos explicativos de interés cuando el sentido de identidad material, sea individual, sea grupal, se usa como título de minuciosas descripciones narrativas. Así, la historia de un individuo o de un grupo indudablemente posee valor explicativo, en particular, insisto, si se trata de una historia específica y detallada. De lo contrario, se cae con facilidad en explicaciones por cliché, meros prejuicios que se ofrecen en lugar del necesario aclarar. Se conocen tales pseudo-explicaciones, especialmente las que apelan a la identidad grupal: un individuo $P$ hizo $x$ porque es... "obrero», «mujer», whomosexual», "negro", clatinoamericano"... o cualquier otro identificador grupal que se use como elogio o estigma, olvidando que cada uno de los conceptos aludidos refiere a complejas y cambiantes historias, y también a complejos y cambiantes individuos. En cuanto al sentido for- mal de identidad su uso explicativo es casi nulo; es difícil imaginar circunstancias apropiadas en las cuales indicar $« . .$. porque $P$ es una persona» conformaría una explicación relevante, informativa.

Más complicados de evaluar son los usos normativos de los sentidos material y formal de identidad. Con respecto al sentido material, sobre todo grupal, suelen hacerse varias distinciones, entre otras, se distingue entre un sentido defensivo de identidad y un sentido propositivo; también se lleva a cabo la distinción entre el papel que juega la preocupación por la identidad en un grupo «central» y en un grupo "marginal» o «periférico», y se razona que, mientras en los grupos centrales esta preocupación tiende a resultar degenerativa, hasta una agresión, en cambio, en los grupos marginales o periféricos se trata de una preocupación no sólo legítina sina incluso vital para la autoimagen, y por lo tanto para el sentido de auto-respeto del grupo en cuestion. Por esto, en relación, por ejemplo, a los obreros, a las mujeres, a los homosexuales, a los negros..., e incluso en relación a complejas regiones culturales como América Latina se busca subrayar la importancia que posee ocuparse con la propia identidad grupal, esfuerzo que debe conducir a una profunda reafirmación, a un fortalecimiento de sí mismos.

Sin embargo, y por importantes que resulten estas preacupaciones, que a menudo lo son, se debe tener cuidado de los peligros que también conllevan. Así, cuando en relación a una identidad grupal, aparentemente en tanto radical, sc habla de cautenticidad», de "defender nuestra identidad", y de inmediato se agrega: "hay que cultivar nuestros propios valores", "hay que reivindicar nuestro pasado"... es necesario enjuiciar el agregado y preguntarse de qué valores en concreto se trata, qué fragmentos particulares del pasado hay que reivin- 
dicar. Pues defender algo por el solo hecho de que lo considere de mi propiedad, en este caso, de mi tradición, no es ninguna hazaña axiológica, más bien un gesto infantil. Por ejemplo, cualquier latinoamericano que esté dispuesto a argumentar no podrá negarse a reconocer que grandes fragmentos de nuestro pasado son irredimibles, y que muchos de los valores vigentes en vastos sectores de América Latina no admiten la menor defensa racional. De ahí que muchas veces consignas como whay que ser autenticos", "hay que defender nuestra identidad como latinoamericanos», si es que quieren implicar algo valioso, no tienen nada que ver con «cultivar nuestros propios valores" o con «reivindicar nuestro pasados, sino al contrario. En casos como éstos, la palabra "autenticidad» suele funcionar como un imán de errores, entre otros, como una invitación a la identidad defensiva, al conformismo autocentrado, al regodeo de sí mismo o de la propia tradición. Como si pertenecer a un grupo marginal o periférico, como si, en general, pertenecer a um grupo fucse un salvoconducto y no articulase, simplemente, una posición, acaso un punto de partida.

Por otra parte, hay también una sofística de la diferencia: el permiso, o exaltación, de lo diverso suele convertirse en una prohibición de esa misma diversidad, aunque la prohibición ya no se decrete en nombre de una abstracción como el identificador universal, sino desde la identidad de un grupo enceguecido; de esta manera, a menudo, ciertas "militancias de contexto" - movirnientos obreros, feministas, homosexuales, de gente de color, étnicos, latinoamericanos... - acaban enredándose en la trampa particularista.

Estas consideraciones, una vez más, reenvian al sentido formal de identidad, a su insistencia en que lo que vale debe valer para cualquiera. Pero si bien es verdad que un sentido formal puede servirnos para enjuiciar los sentidos materiales, tampoco olvidemos que, a veces, éstos enjuician a aquél. Reitero: en no pocas ocasiones tienen razón los dos ataques contextualistas presentados en contra del identificador formal.

En primer lugar, la defensa de una abstracción ha servido como coartada para cometer las peores injusticias: tanto hablar del Hombre suele ser compatible con un desprecio a los hombres y mujeres bien concretos. No sólo compatible, esas universalidades a menudo aconsejan desconocer y hasta reprimir cualquier diversidad $e$ incluso lo hacen invocando los vocabularios políticos más opuestos. En tiempos recientes, y desde la "tradición de la bajeza», se han atacado con razón lo que podemos llamar "las miserias del humanismo»: se ahueca la vo7, se pone "los ojos en blancon y se defiende al Hombre; en cuanto a los hombres y las mujeres "naturales", "históricos", son detalles con los cuales lo mejor es no ensuciarse las manos. Quiero decir: el discurso humanista nunca ha excluido, entre sus prácticas, ni a la explotación, ni al sexismo, ni a los diversos racismos.

En segundo lugar, identificarse e identificar a partir puramente de un identificador formal no configura una forma rica de identificación, no da pie, en general, a ninguna solidaridad.

No obstante la verdad que contengan estos dos ataques, ello no impide que el identificador universal poscca con frecuencia un valor normativo insustituible en tanto destructor de aquellas obsesiones - no pocas veces delirantes y hasta en armas- a que obligan los cultos de la diferencia y su secuela, los fanatismos de grupo, las complicidades con sus peores excesos, con sus retóricas más suicidas. Por eso, los fenómenos llamados «debilitamiento» y "pérdida de la identidad» son ambiguos. Por 
un lado, suelen resultar una catástrofe, tal vez una aniquilación: la ruina de las narrativas sobre sí mismo, individuales y grupales, y en consecuencia de la integración en un medio social; por otro lado, sin embargo, este debilitamiento o pérdida es posible que también configure una ocasión de cambio, la sustitución de convenciones y valores estrechamente grupales y hasta amenazadores de los otros grupos, por reglas y valores universales, permitiendo de este modo la creación de una identidad, tanto individual como grupal, más compleja, menos rígida, más abarcadora.

Hay, pues, un vértigo de lo sublime que imponicndo una identidad puramente formal conduce de hecho a encubrimientos y vacíos, o más precisamente, al dilema: el identificador formal es encubridor o vacio. Pcro hay también un vértigo de la bajeza en donde la mayor radicalidad no es más que la otra cara del auto-conformismo, y el fervor por lo diverso se convierte en su censor más feroz. Lo que implica la necesidad de tener presente que algunas preguntas por la identidad como "¿quién soy yo?", "¿quién eres tú?", no son meras preguntas referericiales, sino también enfáticamente críticas, y que sus respuestas prácticas - sus respuestas sobre todo morales y políticas- no conforman, no pueden conformar, una perspectiva unitaria: apelizn a un concepto de identidad en conflicto, a un concepto de identidad en tanto concepto normativamente desgarrado. ¿Cómo es esto?

De manera inevitable tenemos una identidad material, más todavía, de manera inevitable tenemos no una, sino varias identidades materiales en tanto pertenecemos a una tradición y a una o varias comuinidades. Sin embargo, específicamente con respecto a los miembros de sociedades no tradicionales, no se puede suprimir la identidad formal sin eliminar una dimensión decisiva de su identidad, dimensión que no en pocas ocasiones introduce ciertas inquietudes turbadoras, a veces muy agudas; éstas, de ser irrazonablemente aquietadas, retrotraen a formas estrechas, agobiantes, degeneradas, de identidad. De ahí que tal vez no sea exagerado afirmar que la identidad formal puede funcionar en relación con las identidades materiales a la manera de una ventana oxigenante: "abre" las identidades materiales, inevitablemente particulares, al horizonte de la universalidad.

Acaso se dude: cuál es el status del desgarramiento, al menos, entre las identidades materiales y la identidad formal? Respuesta "gruesa»: no se trata de un conflicto entre la experiencia vivida - en una comunidad, en una tradicióny la lógica, incluso la lógica trascendental, o algo por el estilo, sino de un desgarre entre puntos de vista igualmente partes de la experiencia vivida. Sólo que mientras los identificadores materiales conforman puntos de vista resultados de encuentros y desencuentros en una o varias comunidades y en una tradicición concretas, y reforzadas cotidianamente por las vivencias en esa o esas comunidades y en esa tradición, el identificador formal, cuando articula un punto de vista efectivo y no vana retórica, parte de un aprendizaje "a contracorriente», una experiencia cuyos materiales son, en alguna medida, abstracciones. No obstante, esas abstracciones pertenecen también a la experiencia: podemos educar una sensibilidad para lo abstracto tanto como educamos una sensibilidad para lo concreto. Así, con la identidad formal se introduce entre nuestras formas de vida, otra forma de vida: la universalidad como forma de vida.

Por todo ello, un miembro de una sociedad no tradicional no puede evitar verse confrontado con la irreductible complejidad de su experiencia, con un - con frecuencia - agitado juego de 
identidades y, a la vez, con el juego de esas identidades materiales con la identidad formal. Insisto, su identidad no puede dejar de estar desgarrada por esa complejidad.

La identidad no tradicional no es, entonces, un dato, sino una búsqueda. Identidades, no identidad, narrativas construidas a partir de requerimientos opuestos: la especie humana como perteneciendo, a la vez, a una clase natural e histónica y a una clase normativa. De esta manera, en las sociedades no tradicionales, habrá argumentaciones donde es necesario defender la universidad formal de la identidad de las personas, y otras en las que lo correcto es precisar la diferencia, las no pocas veces sorprendentes materialidades de nuestras variadas historias, tanto individuales como sociales.

\title{
¿Una pragmatización de la teoría crítica?
}

\author{
(El doble gesto de Thomas McCarthy)
}

\author{
CARLOS THIEBAUT \\ Instituto de Filosofía del CSIC
}

El último libro de Thomas McCarthy, Ideales $e$ ilusiones," tiene una estructura doble que denota, quizá, la compleja ubicación intelectual del autor a caballo de tradiciones y culturas intelectuales diversas entrc las que, como ya es sabido, transita con fluidez. Como el subtítulo de la obra señala, este conjunto de trabajos abarca tanto la discusión de los momentos de "desconstrucción" del proyecto moderno en filosofía, como una "reconstrucción" del mismo. En la primera parte del volumen McCarthy ajusta cuentas con diversas críticas neopragmatistas y postestructuralistas (como las de Rorty, Foucault y Derrida) que se han elevado para desconstruir los programas modernos, especificamente kantianos, desde el horizonte teórico que suministra la teoría crítica en su última versión habermasiana. La segunda parte, reconstructiva, del libro se vuelve hacia esa misma teoría crítica e incluye un conjunto de trabajos en los que el autor revisa ese proyecto habermasiano en al- gunos de sus aspectos centrales, pero sobre todo, en lo que al análisís de la dimensión normativa se refiere. El libro contiene, pues, un doble gesto: reafirma, frente a los críticos, algunos supuestos del programa epistémico y normativo de la modernidad $y$, en segundo lugar y desde dentro, con una estrategia de "crítica inmanente», se enfrenta a la versión que de ese programa ofrece Habermas.

McCarthy muestra en el libro una concepción de la filosofía práctica y de sus tareas que se apoya sobre un doble supuesto que aparece en los diversos momentos de sus reflexiones: en primer lugar, sobre la defensa de los ideales de una sociedad democrática y, en segundo lugar, sobre su concepción de un vínculo fuerte entre la filosofía y las ciencias sociales. Ambos elementos forman parte del legado de la teoría crílica que emparenta este trabajo con el proyecto interdisciplinar de Horkheimer de los años treinta y que, tras el giro linguístico, se desarrolla también en la teoría crítica de 